\title{
ON ASYMPTOTIC BEHAVIOR OF PERTURBED NONLINEAR SYSTEMS
}

\author{
R. E. FENNELL AND T. G. PROCTOR
}

\begin{abstract}
A version of the variation of constants formula for nonlinear systems is used to study the comparative asymptotic behavior of the systems $x^{\prime}=f(t, x)$ and $y^{\prime}=f(t, y)+g(t, y)$.
\end{abstract}

1. Introduction. Marlin and Struble [6] showed the asymptotic behavior of the solutions of a nonlinear system determines the asymptotic behavior of perturbations of the system. This paper is a continuation of this problem. We place hypotheses on the basic nonlinear system and its perturbation analogous to those placed on a linear system and its perturbation by Brauer and Wong [2] and Hallam and Heidel [4]. The basic tools of the investigation are a generalized version of the variation of constants formula, a much used comparison principle and fixed point theorems. The proofs are similar to those given in [2] and [4] for perturbations of a linear system.

Let $\alpha$ be a real number, let $\Omega$ be a region in $R^{n}$, let $f$ and $g$ be continuous functions from $[\alpha, \infty) \times \Omega$ into $R^{n}$ such that $f_{x}(t, x)$ exists and is continuous on $[\alpha, \infty) \times \Omega$ and consider the differential equations

$$
\begin{aligned}
x^{\prime} & =f(t, x), \\
y^{\prime} & =f(t, y)+g(t, y) .
\end{aligned}
$$

Let $x(t, \tau, \gamma)$ denote the solution of (1) passing through $\gamma$ at $t=\tau$, let $\Omega_{1}$ be open and such that $\bar{\Omega}_{1} \subset \Omega$ and assume that for $\gamma$ in $\bar{\Omega}_{1}, \alpha \leqq \tau$, the function $x(t, \tau, \gamma)$ exists for $\alpha \leqq t$. It is known [3] the derivative matrix

$$
\frac{\partial x}{\partial \gamma}(t, \tau, \gamma)=\Phi(t, \tau, \gamma)
$$

Presented to the Society, March 26, 1971; received by the editors February 23, 1971. AMS 1969 subject classifications. Primary 3450.

Key words and phrases. Asymptotic behavior, perturbed nonlinear differential equations, variation of constants, Schauder fixed point theorems, asymptotic equivalence.

(c) American Mathematical Society 1972 
exists, satisfies the variational equation

$\Phi(\tau, \tau, \gamma)=I$, and

$$
z^{\prime}=f_{x}(t, x(t, \tau, \gamma)) z
$$

$$
\frac{\partial x}{\partial \tau}(t, \tau, \gamma)=-\Phi(t, \tau, \gamma) f(\tau, \gamma)
$$

Suppose $w(t, \lambda)$ is a continuous nonnegative function on $[\alpha, \infty) \times R^{+}$, is nondecreasing in $\lambda$, and for some $0<k$ there is a unique solution of

$$
r^{\prime}=w(t, r), \quad r(\alpha)=k .
$$

Further we assume $r(t)$, the solution of (3), exists for $t \geqq \alpha$ and $\lim _{t \rightarrow \infty} r(t)=r_{\infty}$. Notice that if $0 \leqq \lambda<r_{\infty}$ we have $\int_{a}^{\infty} w(t, \lambda) d t<\infty$. This follows since $\int_{\alpha}^{\infty} w(s, r(s)) d s$ exists, so for $t_{1}$ such that $\lambda \leqq r(t)$ for $t \geqq t_{1}$,

$$
0 \leqq \int_{t_{1}}^{\infty} w(s, \lambda) d s \leqq \int_{t_{1}}^{\infty} w(s, r(s)) d s<\infty
$$

2. Main results. Theorem 1 below establishes that corresponding to some solutions of (2) which exist for $t \geqq \alpha$ there is a solution of (1) which is asymptotically similar and Theorem 2 investigates the converse problem.

THEOREM 1. Let $D(t)$ be a continuous nonsingular $n \times n$ matrix for $t \geqq \alpha$ and $\Omega_{2} \subset \Omega_{1}$ be such that

(a) $t \geqq \alpha,|D(t) \gamma| \leqq r_{\infty}$ implies $\gamma$ is in $\Omega_{1}$,

(b) $|\bar{D}(t) \Phi(t, \tau, \gamma) g(t, \gamma)| \leqq w(\tau,|D(\tau) \gamma|)$, for $t$, $\tau$ in $[\alpha, \infty), \gamma$ in $\Omega_{1}$,

(c) $|D(t) x(t, \alpha, \gamma)| \leqq k$, for $t \geqq \alpha, \gamma$ in $\Omega_{2}$.

Then for $\gamma$ in $\Omega_{2}$ there is a solution $y(t), \alpha \leqq t$, of (2) passing through $\gamma$ at $t=\alpha ;$ and for each such solution there is a corresponding solution $x^{*}, \alpha \leqq t$, of (1) such that

$$
\lim _{t \rightarrow \infty} D(t)\left[y(t)-x^{*}(t)\right]=0 .
$$

Proof. It is known [1] that for $\gamma$ in $\Omega_{2}$ the solutions of (2) passing through $\gamma$ at $t=\alpha$ satisfy

$$
y(t)=x(t, \alpha, \gamma)+\int_{\alpha}^{t} \Phi(t, s, y(s)) g(s, y(s)) d s
$$

for all $t$ for which $y(t)$ is in $\Omega_{1}$. We have

$$
|D(t) y(t)| \leqq k+\int_{\alpha}^{t} w(s,|D(s) y(s)|) d s ;
$$

thus by standard inequality theorem $[5$, p. 29]

$$
|D(t) y(t)| \leqq r(t) \leqq r_{\infty} .
$$


By (a) in the hypothesis $y(t)$ is in $\Omega_{1}$; therefore $y$ is defined and satisfies (5) for all $t \geqq \alpha$. Further for $\varepsilon>0$ there is a $T$ such that when $T \leqq t_{1} \leqq t_{2}$ we have $0<r\left(t_{2}\right)-r\left(t_{1}\right)<\varepsilon$. Consequently

$$
\begin{aligned}
\left|\int_{t_{1}}^{t_{2}} D(t) \Phi(t, s, y(s)) g(s, y(s)) d s\right| & \leqq \int_{t_{1}}^{t_{2}} w(s, r(s)) d s \\
& =r\left(t_{2}\right)-r\left(t_{1}\right)<\varepsilon ;
\end{aligned}
$$

therefore $\int_{\alpha}^{\infty} \Phi(t, s, y(s)) g(s, y(s)) d s$ exists uniformly for $t$ in compact intervals. Now

thus

$$
\begin{aligned}
D(t) y(t)= & D(t)\left[x(t, \alpha, \gamma)+\int_{\alpha}^{\infty} \Phi(t, s, y(s)) g(s, y(s)) d s\right] \\
& -D(t) \int_{t}^{\infty} \Phi(t, s, y(s)) g(s, y(s)) d s
\end{aligned}
$$

$$
\begin{array}{r}
\left|D(t)\left[y(t)-x(t, \alpha, \gamma)-\int_{\alpha}^{\infty} \Phi(t, s, y(s)) g(s, y(s)) d s\right]\right| \\
\quad \leqq \int_{t}^{\infty} w(s, r(s)) d s=r_{\infty}-r(t) \rightarrow 0
\end{array}
$$

as $t \rightarrow \infty$. It remains to show

$$
x^{*}(t)=x(t, \alpha, \gamma)+\int_{\alpha}^{\infty} \Phi(t, s, y(s)) g(s, y(s)) d s
$$

is a solution of (1). We have

$$
\begin{aligned}
x(t, T, & y(T))+\int_{\alpha}^{\infty} \Phi(t, s, y(s)) g(s, y(s)) d s-x^{*}(t) \\
& =\int_{\alpha}^{T} \frac{d}{d s} x(t, s, y(s)) d s=\int_{\alpha}^{T} \Phi(t, s, y(s)) g(s, y(s)) d s
\end{aligned}
$$

which implies $\lim _{T \rightarrow \infty} x(t, T, y(T))=x^{*}(t)$ uniformly for $t$ in compact intervals. Also

$$
\begin{aligned}
f\left(t, x^{*}(t)\right)-f(t, & x(t, \alpha, \gamma))=\lim _{T \rightarrow \infty} \int_{\alpha}^{T} \frac{d}{d s}[f(t, x(t, s, y(s)))] d s \\
= & \lim _{T \rightarrow \infty} \int_{\alpha}^{T} f_{x}(t, x(t, s, y(s))) \Phi(t, s, y(s)) g(s, y(s)) d s
\end{aligned}
$$

uniformly for $t$ in compact intervals. Therefore

$$
\begin{aligned}
x^{* \prime}(t) & =f(t, x(t, \alpha, \gamma))+\int_{\alpha}^{\infty} f_{x}(t, x(t, s, y(s))) \Phi(t, s, y(s)) g(s, y(s)) d s \\
& =f\left(t, x^{*}(t)\right) .
\end{aligned}
$$


Theorem 2 below investigates the converse problem to that considered in Theorem 1. In the case $f$ is linear in $x$ this problem [4] amounts to showing there is a solution of the integral equation

$$
y(t)=x(t)-\int_{t}^{\infty} \Phi(t, s, y(s)) g(s, y(s)) d s .
$$

This can be done using the Schauder fixed point theorem for general $f$; however if $f$ is nonlinear it is not readily apparent that such a solution satisfies (2).

THEOREM 2. Let the hypothesis of Theorem 1 hold. Then for any solution $x$ of (1) which exists for $t \geqq \alpha$ and such that $x(\alpha)$ is in $\Omega_{2}$, there is a $t_{1} \geqq \alpha$ and a solution $y$ of (2) for $t \geqq t_{1}$ such that $\lim _{t \rightarrow \infty} D(t)[y(t)-x(t)]=0$.

Proof. We begin by constructing a sequence of solutions of (2) which is uniformly convergent on compact subintervals. Let $0<\sigma<r_{\infty}-k$, choose $t_{1} \geqq \alpha$ so large that $\int_{t_{1}}^{\infty} w(s, \sigma+k) d s<\sigma$; and for any integer $n \geqq t_{1}$ let $\mathscr{F}_{n}$ be the set of continuous functions, $v$, from $\left[t_{1}, n\right]$ into $R^{n}$ satisfying

$$
|v(t)| \leqq|D(t) x(t)|+\sigma \text { for } t_{1} \leqq t \leqq n .
$$

First we show that (2) has a solution $v_{n}$ in $\mathscr{F}_{n}$. Define an operator $S$ on $\mathscr{F}_{n}$ by

$$
S v(t)=D(t) x(t)+\int_{n}^{t} D(t) \Phi\left(t, s, D^{-1}(s) v(s)\right) g\left(s, D^{-1}(s) v(s)\right) d s
$$

for $t_{1} \leqq t \leqq n$. We observe

$$
|S v(t)-D(t) x(t)| \leqq \int_{t}^{n} w(s,|v(s)|) d s \leqq \int_{t}^{n} w(s, \sigma+k) d s \leqq \sigma ;
$$

thus $S$ maps $\mathscr{F}_{n}$ into itself. It is easy to see the hypothesis of the Schauder fixed point theorem holds; thus there is a fixed point of $S$ in $\mathscr{F}_{n}$ which we denote by $v_{n}$. The function $y_{n}$ given by $y_{n}(t)=D^{-1}(t) v_{n}(t)$ satisfies

and since

$$
\begin{aligned}
y_{n}^{\prime}(t)= & f(t, x(t))+g\left(t, y_{n}(t)\right) \\
& +\int_{n}^{t} f_{x}\left(t, x\left(t, s, y_{n}(s)\right)\right) \Phi\left(t, s, y_{n}(s)\right) g\left(s, y_{n}(s)\right) d s ;
\end{aligned}
$$

$$
\begin{gathered}
f\left(t, x\left(t, t, y_{n}(t)\right)\right)-f(t, x(t))=\int_{n}^{t} \frac{d}{d s} f\left(t, x\left(t, s, y_{n}(s)\right)\right) d s \\
=\int_{n}^{t} f_{x}\left(t, s, y_{n}(s)\right) \Phi\left(t, s, y_{n}(s)\right)\left[y_{n}^{\prime}(s)-f\left(s, y_{n}(s)\right) d s\right]
\end{gathered}
$$

we have

$$
\omega(t)=-\int_{n}^{t} f_{x}\left(t, x\left(t, s, y_{n}(s)\right)\right) \Phi\left(t, s, y_{n}(s)\right) \omega(s) d s
$$


where $\omega(t)=y_{n}^{\prime}(t)-f\left(t, y_{n}(t)\right)-g\left(t, y_{n}(t)\right)$. But this implies $\omega=0$ thus $y_{n}$ is a solution of $(2)$ on $\left[t_{1}, n\right]$.

Let $N$ be an integer larger than $t_{1}$ and consider the sequence $v_{n}, n=N$, $N+1, \cdots$, of fixed points obtained above. Clearly $\left|v_{n}(t)\right| \leqq k+\sigma$ for $t_{1} \leqq t \leqq N$ and the sequence $\left\{v_{n}\right\}_{N}^{\infty}$ is equicontinuous on this interval.

By Ascoli's theorem there is a subsequence $\left\{v_{n 1}\right\}$ of the $v_{n}$ 's converging uniformly on $\left[t_{1}, N\right]$. Similarly the sequence $\left\{v_{n 1}\right\}$ is defined on $\left[t_{1}, N+1\right]$ for $n 1 \geqq N+1$ and is equicontinuous on $\left[t_{1}, N+1\right]$ so there is a subsequence of the $v_{n 1}$ 's say $\left\{v_{n 2}\right\}$ converging uniformly on this interval. Clearly on the interval $\left[t_{1}, N\right]$ both subsequences converge to the same limit. Proceeding inductively we define a function $v$ on $\left[t_{1}, \infty\right)$ and a chain of subsequences $\left\{v_{n k}\right\}$ such that $\left\{v_{n k}\right\}$ converges uniformly to $v$ on $\left[t_{1}, N+k\right]$. The sequence $\left\{\bar{v}_{n}\right\}_{1}^{\infty}$, where $\bar{v}_{n}=v_{n n}$, then converges to $v$ uniformly on compact subintervals of $\left[t_{1}, \infty\right)$.

By using hypothesis (b) it is easy to see that

$$
\int_{t}^{\infty} D(t) \Phi\left(t, s, D^{-1}(s) v(s)\right) g\left(s, D^{-1}(s) v(s)\right) d s
$$

exists and that

$$
\begin{aligned}
& \lim _{n \rightarrow \infty} \int_{t}^{n} D(t) \Phi\left(t, s, D^{-1}(s)\right.\left.\bar{v}_{n}(s)\right) g\left(s, D^{-1}(s) \bar{v}_{n}(s)\right) d s \\
&=\int_{t}^{\infty} D(t) \Phi\left(t, s, D^{-1}(s) v(s)\right) g\left(s, D^{-1}(s) v(s)\right) d s .
\end{aligned}
$$

The functions $\bar{y}_{n}(t)=D^{-1}(t) \bar{v}_{n}(t)$ are solutions of (2); consequently $y(t)=D^{-1}(t) v(t)$ is also a solution and we note

$$
D(t)[y(t)-x(t)]=-\int_{t}^{\infty} D(t) \Phi(t, s, y(s)) g(s, y(s)) d s
$$

which vanishes at $t \rightarrow \infty$.

Marlin and Struble [6] consider the case $D(t)=I$ and $\Omega_{1}$ is bounded and convex. By arguments used in the proof of Theorem 2 above it is possible to improve the corresponding theorem in [6].

THEOREM 3. Assume $\Omega_{1}$ is bounded and $x$ is a solution of (1) with values in $\Omega_{1}$ for $t \geqq \alpha$ and without limit points on the boundary of $\Omega_{1}$. Further assume that whenever $z$ is a continuous function from $[\alpha, \infty)$ into $\Omega_{1}$ and $\alpha \leqq t \leqq T$ then

$$
\int_{T}^{\infty}|\Phi(t, s, z(s)) g(s, z(s))| d s \leqq J(T)
$$

where $J(T) \rightarrow 0$ as $T \rightarrow \infty$. Then there is a $t_{1} \geqq \alpha$ and a solution $y$ of (2) for $t \geqq t_{1}$ such that $\lim _{t \rightarrow \infty}[y(t)-x(t)]=0$. 
3. An example. The following example is similar to an example given by Brauer and Wong in [2] and shows that the asymptotic behavior of unbounded solutions can be compared. This feature is not present in the theorems of Marlin and Struble [6]. Let $h$ be a continuous real valued function defined on $[0, \infty) \times R^{2}$ which satisfies

$$
|h(t, u, \dot{u})| \leqq h_{0}(t)|u|^{m}+h_{1}(t)|\dot{u}|^{n},
$$

where $h_{0}$ and $h_{1}$ are continuous functions and $m$ and $n$ are positive numbers with $p=\max \{m, n\}>1$. If

$$
\int_{1}^{\infty} \tau^{m+1} h_{0}(\tau) d \tau<\infty, \quad \int_{1}^{\infty} h_{1}(\tau) d \tau<\infty,
$$

$\alpha$ is large enough and $\max \left\{\left|\gamma_{1}\right|,\left|\gamma_{2}\right|\right\} \leqq \frac{1}{2}$ there is a solution $u$ of

$$
\ddot{u}+e^{-t} \dot{u}^{2}=h(t, u, \dot{u}), \quad u(\alpha)=\gamma_{1}, \quad \dot{u}(\alpha)=\gamma_{2},
$$

$u$ exists for $t \geqq \alpha$ and there is a constant $\delta$ such that $u(t)=\delta t+o(t)$ as $t \rightarrow \infty$. Here we used

$$
\begin{aligned}
D(t) & =\left[\begin{array}{ll}
\frac{1}{3} t & 0 \\
0 & 1
\end{array}\right], \quad \Omega_{1}=\left\{\gamma \text { in } R^{2} ;\left|\gamma_{2}\right|<2\right\}, \\
\Omega_{2} & =\left\{\gamma \text { in } R^{2}: \max \left\{\left|\gamma_{1}\right|,\left|\gamma_{2}\right| \leqq \frac{1}{2}\right\}\right\}, \\
w(t, r) & =\lambda(t) \max \left\{r^{p}, 1\right\}
\end{aligned}
$$

where $\lambda$ is constructed as in [2].

\section{REFERENCES}

1. Fred Brauer, Perturbations of nonlinear systems of differential equations, J. Math. Anal. Appl. 14 (1966), 198-206. MR 33 \#359.

2. Fred Brauer and J. S. W. Wong, On asymptotic behavior of perturbed linear systems, J. Differential Equations 6 (1969), 142-153. MR 39 \#570.

3. W. A. Coppel, Stability and asymptotic behavior of differential equations, Heath, Boston, Mass., 1965. MR 32 \#7875.

4. T. G. Hallam and J. W. Heidel, The asymptotic manifolds of a perturbed linear system of differential equations, Trans. Amer. Math. Soc. 149 (1970), 233-241. MR 41 \#2136.

5. P. Hartman, Ordinary differential equations, Wiley, New York, 1964. MR 30 \#1270.

6. J. A. Marlin and R. A. Struble, Asymptotic equivalence of nonlinear systems, J. Differential Equations 6 (1969), 578-596. MR 40 \#5985.

Department of Mathematics, Clemson University, Clemson, South Carolina 29631 